\title{
A Theoretical Framework for Cost Allocations in a Monopoly System with Application to the Allocation of Transmission Losses
}

\author{
Haoming Liu, Junji Wu, Yun Zou, Fushuan Wen, Yixin Ni, Senior Member, IEEE, and \\ F. F. Wu, Fellow, IEEE
}

\begin{abstract}
In this paper, fundamental characteristics of a general monopolistic service system with a simple structure are first investigated, and a theoretical framework for allocations of common costs is then developed. The concepts of "fairness" and "rationality" are characterized mathematically for the general cost allocation problem. Under the proposed framework, several available allocation methods of transmission losses could be well unified.
\end{abstract}

Index Terms--Monopolistic service system, cost allocation, fairness and rationality axioms, active power losses.

\section{INTRODUCTION}

$\mathrm{P}$ OWER industry restructuring has necessitated the decomposition of the three sectors, i.e., generation, transmission and distribution. In the new market environment, transmission open access (TOA) provides a "fair playing field" for power suppliers to compete in the bid-based operation, and for all participants to access and use the transmission facilities. In order to make sure that the transmission service provided is fair to all participants and existing transmission facilities could be utilized efficiently and reasonably, adequate transmission pricing or appropriate transmission cost allocation is very important. Due to geographical, economic and technical reasons, the transmission system will remain a monopoly in the emerging electricity market environment.

One of the major aspects in transmission pricing or transmission cost allocation is the allocation of transmission losses. In the traditional vertically integrated power industry, transmission losses were not dealt with separately but rather included in the total cost and recovered by averagely allocating the cost to customers. In the electricity market environment, the problem of allocating transmission losses to different participants becomes a very important problem. The

This work was jointly supported by National Natural Science Foundation of China (60074007), and a seeding fund project from Hong Kong University.

Haoming Liu and Junji Wu are with School of Power Engineering, Nanjing University of Science and Technology, Nanjing, China (e-mail: liuhaom@hotmail.com, wji807 (è mail.njust.edu.cn ).

Yun Zou is with Department of Autornation, Nanjing University of Science and Technology, Nanjing, China (e-mail: zouyun (j)jlonline.com)

Fushuan Wen, Yixin Ni and F.F. Wu are with the University of Hong Kong, HK(e-mail: fswen@ere.hku.hk., yxni@eed.hhu.hk, ffwu@eee.hku.hk). well-known nonlinear characteristic of the power flow equations makes the problem of rigorously and accurately allocating the losses, from the viewpoint of mathematics, an impossible task.

Several approximate methods or criteria have recently been proposed. Among them, reference [1], [2] proposed the method based on the square of current, while in [3]-[5] the equal proportional share of current was assumed. Another method is to allocate the cross-losses in proportion to current [2], [6], [7]. Since different simplification assumptions are made in these methods, it is difficult to evaluate which one is better than others. Since it is not possible to find a mathematically solid approach for allocating transmission losses, some generally accepted principles must be followed, such as fairness and rationality. However, it is quite difficult, if not impossible, to rigorously define the so-called fairness and rationality, some generally accepted axioms from sociology and economics disciplines have to be employed for this purpose.

Given this background, it is the objective of this paper to investigate fundamental characteristics of a general monopolistic service system with a simple structure and then based on these to develop a theoretical framework for allocations of common costs. Under this framework, several existing methods for transmission loss allocations could be well unified.

\section{THEORETICAL FOUNDATION}

In this section, a simple model of the monopoly system will be used for illustrating the general characteristics of the cost allocation problem.

Let $S$ be a public server in a general sense, and $X_{k}$ $(k=1,2, \ldots, n)$ is a user of the monopoly system. The total number of users, i.e., $n(1 \leq n \leq N)$, is variable, and this means that all users could enter or quit the system freely. Assume that $x_{k} \geq 0(k=1,2, \ldots, n)$ is the service quantity from server $S$ to user $X_{k}$, and the service capability (such as transmission capacity) is not taken into account. Further, assume that the common cost (such as transmission losses) function $\kappa_{n}(x), x=\left[x_{1}, x_{2}, \ldots, x_{n}\right]^{T} \in R^{n}$ is piecewise continuous.

Without loss of generality, suppose that the unit price of 
cost (losses) is 1 , then numerically $\kappa_{n}(x)$ equals the cost. For simplicity, we define the above system model as $\left\{S, X, \kappa_{n}\right\}$. Let $\{S, X, \kappa]=\cup_{n=1}^{N}\left\{S, X, \kappa_{n}\right\}$. If $S$ is the only and unsubstitutable server to all the users, we say $\{S, X, \kappa\}$ is monopolistic.

\section{A. Basic Characteristics of the Model}

Definition 1: Suppose $x=\left[x_{1}, x_{2}, \ldots, x_{n}\right]^{T} \in R^{n}, x_{k} \geq 0$, $k=1,2, \ldots, n .\{S, X, \kappa\}$ is said to be no different, if the followings hold simultaneously,

1) Identity of service measurement: all $x_{k}, k=1,2, \ldots, n$, have the same property and dimension.

2) User independence: all users are function independent, and this means that there does not exist any nonzero function $\sigma: R^{n} \rightarrow R$ to make $\sigma(x)=0$.

Definition 2: The cost function $\kappa_{n}(x)$ is called regular if

1) Positive definite: the cost function is non-negative:

$$
\kappa_{n}(x) \geq 0, \kappa_{n}(0)=0
$$

2) Symmetry: $\kappa_{n}(x)$ is no different to all users $X_{k}$ $(k=1,2, \ldots, n, 1 \leq n \leq N)$, i.e. the function value remains unchanged if any two elements of vector $x$ are exchanged.

Generally speaking, 1) is mandatory for the cost function, and 2 ) is used to simplify the structure of the cost function.

Definition 3: $\{S, X, \kappa\}$ is said to be a simple service system if it is no different and the cost function $\kappa_{n}(x)$ is regular.

This means that in a simple service system the users are equal with simple cost structures. From now on, all problems to be discussed are based on the simple service system.

Suppose

$$
\operatorname{core}\left[\kappa_{n}(x)\right]=\kappa_{n}(x)-\sum_{k=1}^{n} \kappa_{n}\left(x_{k} e_{k}\right)
$$

where, $e_{k}=[0, \ldots, 0,1,0, \ldots, 0]^{T} \in R^{n}$ is a $n$-dimension vector with the $k$-th element as 1 and all the other elements as zero.

Definition 4: core $\left[\kappa_{n}(x)\right]$ is called an inseparable core of $\kappa_{n}(x) . \kappa_{n}(x)$ is said to be separable if $\operatorname{core}\left[\kappa_{n}(x)\right]=0$.

\section{B. Principles of Cost Allocations and Analysis of the Cost Structure}

The two problems, i.e. cost allocation and cost structure analysis, are of course different but tightly related. An axiom system is built for the cost allocation in the previously defined simple service system from the viewpoint of sociology and economics disciplines.

1) An Axiom System:

Let $Q_{k, n}(x)$ be the cost share allocated to user $X_{k}$, $k=1,2, \ldots, n, 1 \leq n \leq N$, in the simple service system $\left\{S, X, \kappa_{n}\right\}$. A set of fairness and rationality axioms for the cost allocation is presented below [8].
Fairness Axiom I (Validity): All allocated cost shares are non-negative, and the sum of them is equal to the total cost.

$$
Q_{k, n}(x) \geq 0, \sum_{k=1}^{n} Q_{k, n}(x)=\kappa_{n}(x)
$$

Fairness Axiom 2 (No-Use-No-Pay): $Q_{k, n}(x)=0$, if $x_{k}=0$.

Fairness Axiom 3 (Equity): Assume that the cost allocation is usage-based. Mathematically, this means that exchanging arbitrarily two elements $x_{k}, x_{i}$ in vector $x$, the values of $Q_{k . n}(x)$ and $Q_{l, n}(x)(k, l=1,2, \ldots, n, 1 \leq n \leq N)$ should also be exchanged,

$$
\begin{aligned}
& Q_{k, n}\left(x_{1}, x_{2}, \ldots, x_{k}, \ldots, x_{l}, \ldots, x_{n}\right) \\
= & Q_{l, n}\left(x_{1}, x_{2}, \ldots, x_{l}, \ldots, x_{k}, \ldots, x_{n}\right) \\
& Q_{l, n}\left(x_{1}, x_{2}, \ldots, x_{k}, \ldots, x_{l}, \ldots, x_{n}\right) \\
= & Q_{k, n}\left(x_{1}, x_{2}, \ldots, x_{l}, \ldots, x_{k}, \ldots, x_{n}\right)
\end{aligned}
$$

Fairness Axiom 4 (Monotonic increase): The allocated cost is monotonically increasing with the usage, i.e. $Q_{k, n}(x)$ is strictly monotonically increasing with respect to $x_{k}$.

Rationality Axiom 1 (Separability property): Each user pay the cost it incurred naturally in a separable system, i.e. $Q_{k, n}(x)=\varphi\left(x_{k}\right)$, if $\left\{S, X, \kappa_{n}\right\}, 1 \leq n \leq N$ is a separable system.

Rationality Axiom 2 (Simple combination additivity): Any simple combination or division would not influence the system structure, i.e. for any $x_{k}, x_{n+1}, k=1,2, \ldots, n$, $1 \leq n \leq N-1$, we have

$$
\begin{aligned}
& Q_{k, n}\left(x_{1}, x_{2}, \ldots, x_{k-1}, x_{k}+x_{n+1}, x_{k+1}, \ldots, x_{n}\right) \\
= & Q_{k, n+1}\left(x_{1}, x_{2}, \ldots, x_{n+1}\right)+Q_{n+1, n+1}\left(x_{1}, x_{2}, \ldots, x_{n+1}\right)
\end{aligned}
$$

Remark 1: The four fairness axioms ensure that all users be treated equally in cost allocations, while the rationality axioms assure that coalition among users could not lead to more profits for the users concerned.

2) Economics Principle:

We could not obtain a definite allocation method directly from the axioms stated before since the applicable allocation functions are not unique. On the other hand, since there exists a tight relationship between the cost structure and the cost allocation, we have to resort to economics principle for tackling the problem. An allocation mechanism which meets the fairness and rationality axioms is presented here based on the well-known marginal costs.

The marginal cost of user $X_{k}(k=1,2, \ldots, n, 1 \leq n \leq N)$ is,

$$
\lambda_{k, n}(x)=\partial \kappa_{n}(x) / \partial x_{k}
$$

A small increment of $\kappa_{n}(x)$ could be expressed as

$$
d \kappa_{n}(x)=\sum_{k=1}^{n}\left[\partial \kappa_{n}(x) / \partial x_{k}\right] d x_{k}
$$

3) Methodology:

Before applying the above procedure to cost allocations, we have to first determine how to deal with the cost structure and the production process of costs concerned. 
Global Principle: All the costs $\kappa_{n}(x)$ are to be allocated.

Local Principle: Only the inseparable core, i.e. $\operatorname{core}\left[\kappa_{n}(x)\right]$, is to be allocated, and the separable parts are allocated directly.

Static principle: The costs to be allocated only relate to the final result $\kappa_{n}[x(T)]$, and not to the intermediate result $\kappa_{n}[x(t)], t \in[0, T]$.

Dynamic Principle: The costs to be allocated relate to not only the final result $\kappa_{n}[x(T)]$, but also the intermediate result $\kappa_{n}[x(t)], t \in[0, T]$.

\section{A THEORETICAL FRAMEWORK FOR COST ALLOCATION IN THE SIMPLE SERVICE SYSTEM}

Based on the work of previous sections, a method based on marginal losses is presented here for allocation of transmission network losses. Suppose that the cost function of each user is monotonically increasing, i.e., for any $k=1,2, \ldots, n, 1 \leq n \leq N$, the function $\lambda_{k, n}(x)=\partial \kappa_{n}(x) / \partial x_{k}$ is non-negative. For simplicity, $\lambda_{k, n}(x)$ is sometimes abbreviated as $\lambda_{k}$ or $\lambda_{k}(x)$. For any $\left\{S, X, \kappa_{n}\right\}, 1 \leq n \leq N$, we have

$$
\begin{aligned}
& \alpha(p)=\left[\begin{array}{lll}
p_{1} / x_{1} & \cdots & p_{n} / x_{n}
\end{array}\right]^{T} \\
& \beta(p)=\left[\begin{array}{lll}
p_{1} / \lambda_{1} & \cdots & p_{n} / \lambda_{n}
\end{array}\right]^{T}
\end{aligned}
$$

where $\lambda_{k}$ is the marginal cost of user $X_{k}$, and $p_{k}=p_{k}(x)$ is the allocated share of user $X_{k}, p=\left[p_{1} \cdots p_{n}\right]^{T}$, $k=1,2, \ldots, n$.

Eq. (8) denotes the allocated share for a unit service quantity, and Eq. (9) the ratio to marginal costs. What the users are concerned with most is how to minimize the average costs showed in (8), and this means that the norm of the vector in (8) should be minimized. From the marginal cost principle, it is known that the norm of the vector in (9) should also be minimized. To take into account of these two requirements, a tradeoff has to be made. Let us consider the following programming problem,

$$
\begin{array}{ll}
\min & \left|\alpha^{T}(p) \beta(p)\right| \\
\text { s.t. } & \sum_{k=1}^{n} p_{k}=1, p_{k} \geq 0
\end{array}
$$

This implies

$$
\lambda_{1}(x)\left[x_{1} / p_{1}(x)\right]=\lambda_{2}(x)\left[x_{2} / p_{2}(x)\right]=\cdots=\lambda_{n}(x)\left[x_{n} / p_{n}(x)\right]
$$

Eq. (11) indicates that the ratio is allocated based on the service quantity if the marginal costs are taken as the penalty factor.

There are two important and useful lemmas as stated below.

Lemma 1: Suppose $\{S, X, \kappa\}$ is a simple service system, then

1) There exists a function $\varphi(\bar{x}), \bar{x}$ is a scalar, for any $1 \leq k \leq n, 1 \leq n \leq N$, we have

$$
\kappa_{n}\left(\bar{x} e_{k}\right)=\varphi(\bar{x})
$$

here, $e_{k}=[0, \ldots, 0,1,0, \ldots, 0]^{T} \in R^{n}$ is a $n$-dimension vector with the $k$-th element as 1 and the others as zero.

2) The inseparable core, i.e., core $\left[\kappa_{n}(x)\right]$, is symmetric, this means that the function value will remain unchanged if any two elements in vector $x$ are exchanged.

Proof: According to the symmetry of the cost structure, for any $1 \leq k, l \leq n, k \neq l$, we have $\kappa_{n}\left(\bar{x} e_{k}\right)=\kappa_{n}\left(\bar{x} e_{l}\right)$, therefore (12) holds. Then

$$
\operatorname{core}\left[\kappa_{n}(x)\right]=\kappa_{n}(x)-\sum_{k=1}^{n} \varphi\left(\bar{x}_{k}\right)
$$

is symmetric. $\square$

Lemma 2: Suppose $\{S, X, \kappa\}$ is a simple service system, if $\lambda_{k, n}(x)=\partial \kappa_{n}(x) / \partial x_{k}$, then $\lambda_{k, n}(x)$ is an equivalent function, i.e. for any $1 \leq k, l \leq n, k \neq l$, we have

$$
\begin{aligned}
& \lambda_{k, n}\left(x_{1}, \ldots, x_{k}, \ldots, x_{l}, \ldots, x_{n}\right)=\lambda_{l, n}\left(x_{1}, \ldots, x_{l}, \ldots, x_{k}, \ldots, x_{n}\right) \\
& \lambda_{l, n}\left(x_{1}, \ldots, x_{k}, \ldots, x_{l}, \ldots, x_{n}\right)=\lambda_{k, n}\left(x_{1}, \ldots, x_{l}, \ldots, x_{k}, \ldots, x_{n}\right)
\end{aligned}
$$

Proof: According to the symmetry of the cost structure, we have

$$
\begin{aligned}
& \lambda_{k, n}\left(x_{1}, \ldots, x_{k}, \ldots, x_{l}, \ldots, x_{n}\right) \\
= & \lim _{\Delta \rightarrow 0}\left\{\left[\kappa_{n}\left(x_{1}, \ldots, x_{k}+\Delta, \ldots, x_{l}, \ldots, x_{n}\right)\right.\right. \\
& \left.\left.-\kappa_{n}\left(x_{1}, \ldots, x_{k}, \ldots, x_{l}, \ldots, x_{n}\right)\right] / \Delta\right\} \\
= & \lim _{\Delta \rightarrow 0}\left\{\left[\kappa_{n}\left(x_{1}, \ldots, x_{l}, \ldots, x_{k}+\Delta, \ldots, x_{n}\right)\right.\right. \\
& \left.\left.-\kappa_{n}\left(x_{1}, \ldots, x_{l}, \ldots, x_{k}, \ldots, x_{n}\right)\right] / \Delta\right\} \\
= & \lambda_{l, n}\left(x_{1}, \ldots, x_{l}, \ldots, x_{k}, \ldots, x_{n}\right)
\end{aligned}
$$

It should be mentioned that the equivalent property in lemma 2 and the symmetry in definition 2 are completely different.

\section{A. Static Method}

From (11), we have,

$$
p_{k}(x)=\left[x_{k} \lambda_{k}(x)\right] / \sum_{l=1}^{n}\left[x_{l} \lambda_{l}(x)\right]
$$

So a static global allocation method could be formulated as

$$
Q_{k, n}(x)=\left[x_{k} \lambda_{k}(x) \kappa_{n}(x)\right] / \sum_{i=1}^{n}\left[x_{l} \lambda_{l}(x)\right]
$$

and a static local allocation method as

$$
Q_{k, n}(x)=\left\{x_{k} \lambda_{k}(x) \operatorname{core}\left[\kappa_{n}(x)\right]\right\} / \sum_{i=1}^{n}\left[x_{l} \lambda_{l}(x)\right]+\varphi\left(x_{k}\right)
$$

$\varphi\left(x_{k}\right)$ is already defined in (12).

Theorem 1: Suppose $\{S, X, \kappa\}$ is a gaining type simple service system, then

1) The static global allocation method as shown in (14) satisfies all the fairness axioms. 
2) The static local allocation method as shown in (15) satisfies not only all the fairness axioms, but also the separability property rationality axiom.

The proof procedure is omitted here due to the space limitation.

\section{B. Dynamic Method}

To obtain the allocation result in view of the dynamic property, we can calculate an incremental quantity first, and then sum them up. From (7), the increment function in time $t$ is,

$$
d \kappa_{n}[x(t)]=\sum_{k=1}^{n}\left\{\partial \kappa_{n}[x(t)] / \partial x_{k}(t)\right\} \dot{x}_{k}(t) d t
$$

which marginal function is the same as that in (6).

From (13) to (15), we can obtain the dynamic global allocation method as follow,

$$
\begin{aligned}
& Q_{k, n}(x)= \\
& \int_{0}^{T}\left\{x_{k}(t) \lambda_{k}[x(t)] \sum_{l=1}^{n}\left\{\left[\partial \kappa_{n}(x(t)) / \partial x_{l}(t)\right] \dot{x}_{l}(t)\right\} / \sum_{l=1}^{n}\left\{x_{l}(t) \lambda_{l}[x(t)]\right\}\right\} d t
\end{aligned}
$$

and the dynamic local allocation method,

$$
\begin{aligned}
Q_{k, n}(x)= & \int_{0}^{T}\left\{x_{k}(t) \lambda_{k}[x(t)] \sum_{l=\}}^{n}\left\{\left[\partial \operatorname{core}\left[\kappa_{n}(x(t))\right] / \partial x_{l}(t)\right] \dot{x}_{l}(t)\right\}\right. \\
& \left./ \sum_{l=1}^{n}\left\{x_{l}(t) \lambda_{l}[x(t)]\right\}\right\} d t+\int_{0}^{T} \dot{\varphi}\left[x_{k}(t)\right] d t
\end{aligned}
$$

where, $\dot{\varphi}\left[x_{k}(t)\right]$ is the separable allocated share to user $X_{k}$ in time $t$.

Theorem 2 (Dynamic fairness theorem): Suppose $\{S, X, \boldsymbol{x}\}$ is a gaining type simple service system, and the marginal cost functions of all users are monotonically increasing, i.e. $\lambda_{k, n}(x)=\partial \kappa_{n}(x) / \partial x_{k}$ is the non-negative monotonically increasing function of $x_{k}$ for any $k=1,2, \ldots, n, 1 \leq n \leq N$. Then

1) The dynamic global allocation method as defined in (16) satisfies all the fairness axioms.

2) The dynamic local allocation method as defined in (17) satisfies not only all the fairness axioms, but also the separability property rationality axiom.

The proof procedure is omitted here due to the space limitation.

\section{Allocation OF Active POWER LoSSES IN TRANSMission SYSTEMS}

Several different allocation methods for active power losses in transmission systems.could be obtained by applying the theoretical framework developed before. Specific to this problem, $S$ is TRANSCO, $\kappa_{n}(x)$ is the active power losses in a given branch $m, x_{k}(k=1, \cdots, n)$ is the current $I_{k}$ of another branch $k$ connecting with branch $m$. Suppose that all transmission losses are undertaken by consumers, the problem is how to allocate the active losses of branch $m$ to all users concerned. We have

$$
\kappa_{n}(x)=C\left(\sum_{k=1}^{n} x_{k}\right)^{2}
$$

here, $C$ is a constant.

\section{A. Proportional Share on Total Losses}

If the static global allocation method is employed, from (14) and (18), we have

$$
\begin{aligned}
& Q_{k, n}(x)=\frac{\left[x_{k} \lambda_{k}(x) k_{n}(x)\right]}{\sum_{l=1}^{n}\left[x_{l} \lambda_{l}(x)\right]} \\
& Q_{j, n}(x)=\frac{\left[x_{j} \lambda_{j}(x) k_{n}(x)\right]}{\sum_{l=1}^{n}\left[x_{l} \lambda_{l}(x)\right]}
\end{aligned}
$$

where

$$
\lambda_{k}(x)=\lambda_{j}(x)=\frac{\partial \kappa_{n}(x)}{\partial x_{k}}=\frac{\partial \kappa_{n}(x)}{\partial x_{j}}=2 C \sum_{i=1}^{n} x_{l}
$$

and then we have

$$
\frac{Q_{k, n}(x)}{Q_{j, n}(x)}=\frac{x_{k} \lambda_{k}(x)}{x_{j} \lambda_{j}(x)}=\frac{x_{k}}{x_{j}}
$$

The allocated shares are proportional to currents, and the same conclusion was obtained in [3] and [4]. This allocation method satisfies all fairness axioms, and simple combination additivity axiom because of $\lambda_{k}(x)=\lambda_{j}(x)=2 C \sum_{i=1}^{n} x_{l}$.

\section{B. Proportional Share on Inseparable Core}

Similarly, from (15) and (18), by following the static local allocation method we have

$$
\begin{aligned}
& Q_{k, n}(x)=\frac{x_{k} \lambda_{k}(x) \operatorname{core}\left[k_{n}(x)\right]}{\sum_{l=1}^{n}\left[x_{l} \lambda_{l}(x)\right]}+\varphi\left(x_{k}\right)=Q_{k, n}^{\text {core }}(x)+\varphi\left(x_{k}\right) \\
& Q_{j, n}(x)=\frac{x_{j} \lambda_{j}(x) \operatorname{core}\left[k_{n}(x)\right]}{\sum_{l=1}^{n}\left[x_{i} \lambda_{l}(x)\right]}+\varphi\left(x_{j}\right)=Q_{j, n}^{\text {core }}(x)+\varphi\left(x_{j}\right)
\end{aligned}
$$

here, $\lambda_{k}(x)=\lambda_{j}(x)=\frac{\partial \kappa_{n}(x)}{\partial x_{k}}=\frac{\partial \kappa_{n}(x)}{\partial x_{j}}=2 C \sum_{i=1}^{n} x_{l}$

and then $\frac{Q_{k, n}^{\text {core }}(x)}{Q_{j, n}^{\text {core }}(x)}=\frac{x_{k} \lambda_{k}(x)}{x_{j} \lambda_{j}(x)}=\frac{x_{k}}{x_{j}}$

In this case the allocated shares are proportional to the currents in the inseparable core, and the separable parts could be allocated to users directly. The same conclusion could be obtained in [6] and [7]. This allocation method satisfies all fairness and rationality axioms.

\section{Allocation of Losses Based on the Square of Current}

If $\frac{\lambda_{k}(x)}{\lambda_{j}(x)}=\frac{x_{k}}{x_{j}}, \lambda_{i}=\partial\left(C x_{i}^{2}\right) / \partial x_{i}=2 C x_{i}$, we can conclude that the allocation method is based on the square of current using either the global method or the local method. Hence, 
based on the theoretical framework developed, we can get the same conclusion as that in [1] and [2].

From the above results, it could be concluded that the developed theoretical framework could well unify several available transmission loss allocation methods.

\section{CONCLUDING REMARKS}

In this work, a theoretical framework for cost allocation in a general monopoly system is developed from the viewpoints of sociology and economics. Several fairness and rationality axioms are presented. Based on the framework, several different allocation principles are proposed and discussed. Finally, the problem of allocating active power losses in transmission systems is investigated, and it is concluded that several existing transmission loss allocation methods could be well unified in the proposed theoretical framework.

\section{REFERENCES}

[1] C. N. Macqueen, and M. R. Irving, "An algorithm for the allocation of distribution system demand and energy losses," IEEE Trans. Power System, vol. 11, pp. 338-343, Feb. 1996.

[2] Antonio Gómez Expósito, Jesús Manuel Riquelme Santos, Tomás González García, and Enrique A. Ruiz Velasco, "Fair allocation of transmission power losses," IEEE Trans. Power System, vol. 15, pp. 184188, Feb. 2000.

[3] Wang Xifan, Wang Xiuli, and Jia Bin, "Fundament of loadflow analysis in wheeling costing - loss allocation problem," Electric Power, vol. 31, pp. 6-9, 1998.

[4] R. Reta, and A. Vargas, "Electricity tracing and loss allocation methods based on electric concepts," IEE Proc,-gener. Transm. Distrib., pp. 518522, Jun. 2001.

[5] J. Bialek, "Tracing the flow of electricity," IEE Proc.-Gener. Transm. Distrib., vol. 143, pp. 313-320, Jul. 1996.

[6] Zuwei Yu. "The proposed CLP method for allocating real power losses of multiple flows," IEEE Power Engineering Review, pp. 45-47, Nov. 2001.

[7] Li Weidong, Sun Hui, and Zhao Shanjun, "Research on rigorous mathematical derivation of loss allocation rule in transmission cost calculation," Automation of Electric Power Systems, vol. 25, pp. 32-35, Jun. 2001.

[8] Qi Huan, Modeling Method of Mathematics, Wuhan: HUST Press, China. 1996. p. 98

\section{BIOGRAPHIES}

Haoming Liu was born in Jiangsu China, on Feb, 1977. He received his B. Sc. and $\mathbf{M}$. Sc. in Electrical Engineering from Nanjing University of Science and Technology (NUST), China, in 1998 and 2001 respectively. He is now pursuing his Ph. D. in Power Engineering School of NUST. His current research interest is focused on electricity market.

Junji Wu is a professor of Power Engineering school at NUST. He received his BE and ME degrees from, NUST, China, in 1982 and 1992 respectively. His current research interests are power system planning, power market and nonelectric measuring.

Yun Zou is a professor of Department of Automation at NUST. His current research interests are control theory and control engineering, power system stability and electricity market.

Fushuan Wen received his BE and ME degrees from Tianjin University, China, in 1985 and 1988. respectively, and $\mathrm{PhD}$ from Zhejiang University, China, in 1991, all in electrical engineering. He joined the faculty of Zhejiang Unversity, China, in 1991, and has been professor there since 1997. He held a visiting position in the National University of Singapore from 1995 to 1997, and was a research fellow in the Hong Kong Polytechnic University from July 1998 to February 2001 . He is now a research assistant professor in the University of
Hong Kong. His current research interests are in power industry restructuring and artificial intelligence applications in power systems.

Yixin Ni (SM'94) received her B. Eng., M. Eng., and Dr. Eng. all from Electrical Engineering Department, Tsinghua University, P.R. China in 1968, 1981 and 1983 respectively. Her research interests are in power system modeling, simulation, stability and control, and power electronics applications in power systems. She was a professor of Tsinghua University and is now with the University of Hong Kong. She is a senior member of CSEE and Fellow of HKIE.

Felix F. Wu (M'73, SM'86, F'89) received his Ph. D. from University of California at Berkeley (UCB). He is now a chair professor of electrical engineering at the University of Hong Kong. Prior to that he was a professor and vice chairman of Dept. of EECS, UCB. His research interests are in power industry restructuring, power system investment planning, design of modern control centers, distribution automation, distributed processing etc. 